\title{
Two-year longitudinal evaluation of a second-generation thin-strut sirolimus-eluting bioresorbable coronary scaffold with hybrid cell design in porcine coronary arteries
}

\author{
Pawel Gasior ${ }^{1,2}$, Yanping Cheng ${ }^{1}$, Jinggang Xia ${ }^{1}$, Gerard B. Conditt ${ }^{1}$, \\ Jennifer C. McGregor ${ }^{1}$, Renu Virmani ${ }^{3}$, Juan F. Granada ${ }^{1}$, Grzegorz L. Kaluza ${ }^{1}$ \\ ${ }^{1}$ CRF-Skirball Center for Innovation, Orangeburg, NY, United States \\ ${ }^{2}$ Division of Cardiology and Structural Heart Diseases, Medical University of Silesia, Katowice, Poland \\ ${ }^{3}$ CVPath Institute, Gaithersburg, MD, United States
}

\begin{abstract}
Background: The first commercially available bioresorbable scaffold (BRS) had a strut thickness of 156 microns. As such, it had the potential for delivery challenges and higher thrombogenicity. The aim herein, is to evaluate biomechanical performance, pharmacokinetics and vascular healing of a novel thin strut (100 $\mu \mathrm{m}$ ) sirolimus eluting BRS (MeRes-100, Meril Life Sciences, Gujarat, India) against the once clinically used BRS (Absorb BVS, Abbott, Santa Clara, CA) in porcine coronary arteries.

Methods: Following device implantation, angiographic and optical coherence tomography (OCT) evaluation were performed at 45, 90, 180 days, 1 year and 2 years. Histological evaluation was performed at 30, 90 and 180 days.

Results: At 2 years, both lumen (MeRes-100 $7.07 \pm 1.82 \mathrm{~mm}^{2}$ vs. Absorb BVS $7.57 \pm 1.39 \mathrm{~mm}^{2}, p=N S$ ) and scaffold areas (MeRes-100 $9.73 \pm 1.80 \mathrm{~mm}^{2}$ vs. Absorb BVS $9.67 \pm 1.25 \mathrm{~mm}^{2}, p=N S$ ) were comparable between tested and control scaffolds. Also, the late lumen area gain at 2 years was similar in both groups tested (MeRes-100 $1.03 \pm 1.98 \mathrm{~mm}^{2}$ vs. Absorb BVS $0.85 \pm 1.56 \mathrm{~mm}^{2}, p=N S$ ). Histologic examination up to 6 months showed comparable healing and inflammation profiles for both devices.

Conclusions: The novel sirolimus-eluting BRS with thinner struts and hybrid cell design showed similar biomechanical durability and equivalent inhibition of neointimal proliferation when compared to the first-ever Absorb BVS up to 2 years in normal porcine coronary arteries. (Cardiol J 2020; 27, 2: 115-125)
\end{abstract}

Key words: optical coherence tomography, bioresobable scaffolds, histology

\section{Introduction}

Bioresorbable scaffolds (BRS) acting as a transient mechanical support during the arterial restorative process had been hoped to provide additional benefits over metallic drug-eluting stents (DES), such as restoration of the treated arterial segments to a more physiological state via return of vasomotion and elasticity, and eventually late lumen gain [1], translating into tangible reduction of long-term adverse effects of percutaneous coronary revascularization $[2,3]$. However, the recently published 2-3-year results from ABSORB II and III trials, have proven otherwise, demonstrating an opposite trend toward higher adverse events in patients treated with the everolimus-eluting Absorb BVS $[4,5]$. Since a majority of these untoward effects have been noted in small coronary

Address for correspondence: Grzegorz L. Kaluza, MD, PhD, CRF Skirball Center for Innovation, 8 Corporate Dr., Orangeburg, NY, 10965, USA, tel: (845) 580 3100, fax: (845) 359 3082, e-mail: gkaluza@crf.org

Received: 27.03.2018 Accepted: 30.06.2018 


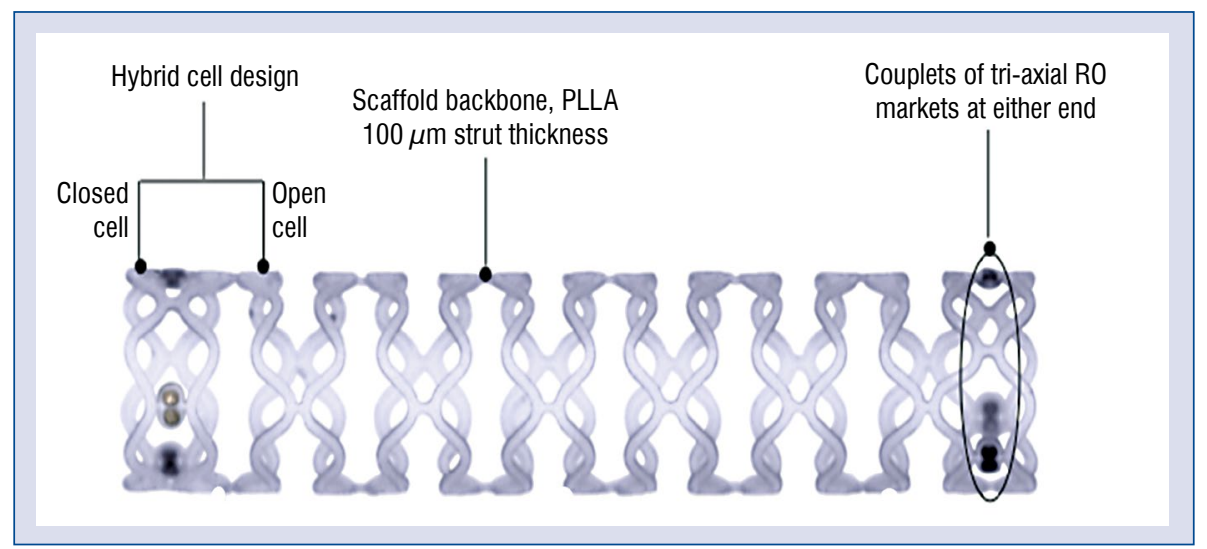

Figure 1. Closed cell-open cell hybrid architecture of MeRes-100 scaffold. The scaffold backbone design features a repeated, alternatingly oriented zigzag crowns connected by longitudinal links with a hybrid cell design comprised of a mix of closed cells at the edges and open cells along the length in order to provide optimal radial strength and uncompromised side branch access (open cells in the middle) without worsening flexibility, allowing strut thickness of $\sim 100 \mu \mathrm{m}$; PLLA — poly-L-lactic acid.

arteries, it is believed that the bulky strut thickness of $156 \mu \mathrm{m}$, exceeding nearly twice that of a contemporary metallic DES, is responsible for poorer deliverability and higher thrombotic and restenotic potential of this technology [6]. It is known that a correlation between strut thickness and flow disturbance exists and translates into negative clinical consequences, such as higher restenosis and target vessel revascularization rates [7]. Large strut thickness also increases flow disturbances with separation, stagnation, and reattachment of blood elements, which may affect the risk of thrombosis [8]. It is therefore imperative for the mainstream success of BRS that the strut thickness be reduced, resulting in no adverse effect on biomechanics.

In this particular study, the aim was to evaluate such an example by assessing biomechanical performance, pharmacokinetics and vascular healing of a novel thin strut sirolimus-eluting poly-L-lactic acid (PLLA) based BRS with hybrid cell design (MeRes-100, Meril Life Sciences, Gujarat, India) in comparison to the Absorb BVS (version 1.1, Abbott Vascular, Santa Clara, CA).

\section{Methods}

\section{Device description}

The MeRes-100 is a thin strut $(\sim 100 \mu \mathrm{m})$ balloon expandable bioresorbable scaffold comprised of a PLLA backbone and topcoat of poly-DL-lactic acid (PDLLA) and sirolimus at the 1:1 ratio resulting in $1.25 \mu \mathrm{g} / \mathrm{mm}^{2}$ (Fig. 1). In the current study, thin strut MeRes-100 BRS was compared to the once commercially available everolimus eluting Absorb BVS with strut thickness of $156 \mu \mathrm{m}$. The device sizes used in the study were as follows: MeRes-100 $(3.0 \times 13,3.5 \times 13,3.0 \times 18$ and $3.5 \times 18 \mathrm{~mm}$ ) and Absorb (sizes $3.0 \times 12,3.5 \times 12$, $3.0 \times 18$ and $3.5 \times 18 \mathrm{~mm}$ ).

\section{In vivo biomechanical performance} and healing studies

The Institutional Animal Care and Use Committee approved this study, and all animals received care in accordance with the Guide for the Care and Use of Laboratory Animals.

To evaluate the healing response of the MeRes-100 in direct comparison to Absorb BVS, histological analysis was performed in a group of 6 young adult Yucatan minipigs sacrificed at 30, 90 and 180 days. Therefore, in total 9 MeRes-100 $(\mathrm{n}=3$ per timepoint $)$ and 6 Absorb $(\mathrm{n}=2$ per timepoint) scaffolds were implanted in 6 animals among all three coronary arteries at the overstretch ratio of 1.1:1, and the implant location was determined by the size of coronary arteries. Mean vessel diameters were measured by quantitative coronary analysis to guide sizing of the devices.

Long term biomechanical performance using serial angiography and optical coherence tomography (OCT) measurements was evaluated in a second group of 5 young adult Yucatan minipigs in which a total of $n=9$ MeRes-100 and $n=4 \mathrm{Ab}$ sorb BVS were implanted. The continuous imaging was performed in all animals immediately after implantation, at 45, 90, 180 days, 1 and 2 years. All 5 animals survived beyond 2 years for ongoing 
imaging evaluation The device implantation was performed with standard percutaneous technique utilizing heparin for anticoagulation. Oral clopidogrel and acetylsalicylic acid was initiated the day prior to the procedure and were maintained throughout the study.

\section{In vivo pharmacokinetic study}

The pharmacokinetic profile of MeRes-100 $(\mathrm{n}=10)$ at 30,90 and 180 days was evaluated using the liquid chromatography mass spectrometry (LCMS/MS), method on file at Agilux Labs (Natick, Massachusetts). Briefly, scaffolds were extracted in organic solvents. Prior to extraction, tissue samples were prepared by dicing followed by homogenization in buffer. Sirolimus in the arterial tissue extracts was analyzed by LC-MS/MS using ascomycin as the internal standard. Quantitation was performed versus a calibration curve and the lower limit of quantitation was $50 \mathrm{ng} / \mathrm{mL}$. Sirolimus was analyzed in whole blood using protein precipitation followed by a solid phase extraction procedure and analysis by LC-MS/MS. Quantitation was performed versus a calibration curve and the lower limit of quantitation was $0.500 \mathrm{ng} / \mathrm{mL}$. The percentage of drug released from the scaffold at each time point was determined by measuring the residual total content on the explanted stents. The sirolimus concentration in whole blood was evaluated in 6 pigs bearing $17 \mathrm{MeRes}-100$ scaffolds. Blood samples were collected at 1, $30 \mathrm{~min}, 1,2$, 4, 8, $24 \mathrm{~h}$ and 7, 14, 28, 60, 90 days post implant. Pharmacokinetic analysis of data on the sirolimus blood concentration over time was performed using the noncompartmental model of Phoenix WinNonlin Version 6.3 software (Pharsight Corporation, St. Louis, MO). The only commercially available BRS at the time of the study initiation was the everolimus-eluting Absorb BVS. Therefore, due to a lack of the device eluting the same antiproliferative agent (sirolimus) to serve as a direct comparator, a control group could not be included for the in vivo pharmacokinetic evaluation.

\section{Quantitative coronary angiography}

Quantitative coronary analysis was performed using QAngio XA SoftwareTM 7.1.14.0 (Medis Medical Imaging System, Leiden, Netherlands). The outer diameter of the contrast-filled catheter was used as the calibration standard and the minimum lumen diameter (MLD) was obtained from the single worst view, while the reference vessel diameter (RVD) was automatically calculated by the interpolation method. The percent diameter stenosis (\%DS) was calculated from the MLD and the RVD. Acute absolute scaffold recoil was defined as the difference between mean diameter of the scaffold delivery balloon at the highest pressure at implantation (X) and mean luminal diameter of stented segment after implantation $(\mathrm{Y})$. Percent acute recoil was calculated as $(\mathrm{X}-\mathrm{Y}) / \mathrm{X} \times 100$ [9].

\section{Optical coherence tomography imaging}

Optical coherence tomography images were recorded using the ILUMIEN PCI Optimization System (St. Jude Medical, St. Paul, MN). Motorized OCT pullbacks were performed at a rate of $20 \mathrm{~mm} / \mathrm{s}$. All images were acquired at 100 frames per second, displayed with a color look-up table and digitally archived. Qualitative and quantitative analyses were performed with the commercial software (ILUMIEN OPTIS, St. Jude Medical, St. Paul, MN) by an experienced analyst blinded to the device information and study methodology, every $1 \mathrm{~mm}$ within the scaffold segment and including $5 \mathrm{~mm}$ of the proximal and distal reference segments. The following cross-sectional parameters were generated as previously described [10]: the lumen area, the inner and outer scaffold area, percentage area of stenosis (\%AS) and neointimal thickness (NIT). For the purpose of this study lumen area gain was calculated as mean lumen area at follow up minus mean lumen area at post-implant. Absolute late recoil was measured as the mean scaffold diameter at post-implant minus the mean scaffold diameter at follow-up. Relative late recoil was calculated as: (absolute late recoil)/post-implant mean scaffold diameter $\times 100$ [11]. Patency ratio was calculated as follow-up lumen area/follow-up reference vessel area, and its changes were also evaluated at different time points. The patency ratio was calculated to determine the relationship between the caliber of the scaffold-treated vessel region versus the caliber of the reference vessel segments proximal and distal to the treated region [12].

\section{Histological analysis}

An independent pathology laboratory (CVPath Institute, Gaithersburg, MD) conducted the histopathologic and histomorphometric analysis. Hearts were removed immediately following termination and the coronary arteries were pressure fixed with $10 \%$ neutral buffered formalin. Treated vessels were cut at three in-scaffold levels (proximal, mid, distal) and two reference vessel levels. All vessel segments were cut twice serially at $\sim 5$ microns and stained with hematoxylin and eosin and elastin trichrome. The resulting slides were examined via 


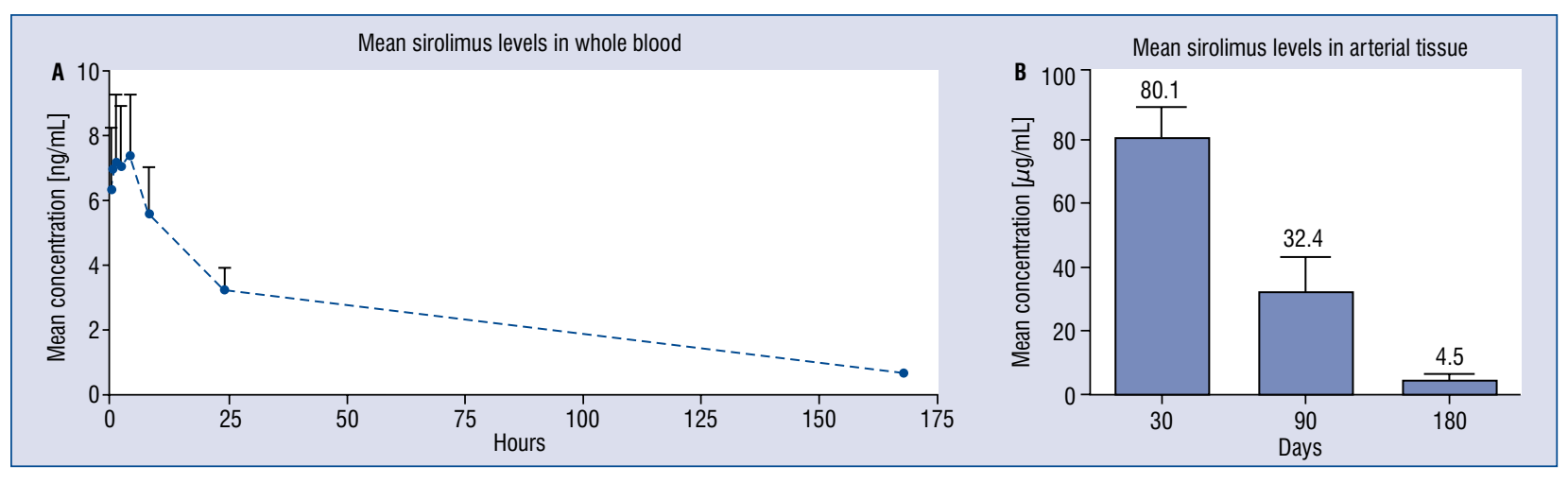

Figure 2. Pharmacokinetic data showing sirolimus release profile (A) sirolimus in whole blood over time (B) sirolimus concentrations in the arterial tissue at different time intervals after MeRes-100 bioresorbable scaffolds implantation.

light microscopy. The cross-sectional areas delineated by external elastic lamina (EEL), internal elastic lamina (IEL) and lumen were measured in each section. The neointimal thickness was defined as the distance from the inner surface of each strut to the luminal border. Neointimal area was defined as the difference of IEL area minus lumen area. Percentage area of stenosis $(\% \mathrm{AS})=[1-$ (lumen area/IEL area) $\times 100$. Qualitative histological assessment on inflammation and healing was performed using a standardized score system [13]. Only 9 MeRes-100 and 6 Absorb BVS underwent histologic examination in this study.

\section{Definitions}

The biomechanical performance endpoint included angiographic (MLD, \%DS, absolute and percent scaffold recoil), OCT (lumen area, scaffold area, neointimal area, \%AS, NIT, late lumen gain, absolute and relative scaffold recoil) and histomorphometric parameters (lumen area, EEL, IEL, NIA, NIT, \%AS). Biocompatibility evaluation comprised of qualitative histological assessment (uncovered struts, neoinitma inflammation, adventitia inflammation, fibrin deposition). Pharmacokinetic evaluation consisted in measuring sirolimus concentration in whole blood and arterial tissue at different time intervals.

\section{Statistical analyses}

Statistical analyses was performed using SAS statistical software (version 9.4; SAS Institute Inc. $\mathrm{NC}$ ). All measurements were expressed as mean \pm standard deviation. Values were compared between groups using non-parametric Mann-Whitney $\mathrm{U}$ tests. The change in continuous outcomes over 5 time periods (30, 90, 180 days, 1 and 2 years) was compared between the two treatment groups using analysis of variance (ANOVA) for repeated measurements. Post-hoc comparisons were performed using the Scheffe test. The differences were considered significant only if the calculated $\mathrm{p}$ value was $<0.05$.

\section{Results}

\section{Pharmacokinetic study}

Results of the pharmacokinetic analysis are summarized in Figure 2. The sirolimus arterial tissue concentrations gradually declined over time, at 92 days they were about $60 \%$ lower than at 30 days and at 186 days were still detectable yet about $90 \%$ lower than at 30 days (Fig. 2A). The results of the sirolimus concentration in whole blood are shown in Figure 2B. Peak drug concentrations in blood were observed at 1-4 h post implant and the average peak concentration of sirolimus was $7.38 \pm 0.42 \mathrm{ng} / \mathrm{mL}$. The blood levels of sirolimus quickly declined to below the limit of quantification after 14 days.

\section{In vivo study of arterial responses}

Quantitative coronary angiography analysis. Before injury, the average vessel diameters were comparable among both groups (MLD: MeRes-100 $2.55 \pm 0.34 \mathrm{~mm}$ vs. Absorb BVS $2.62 \pm 0.29 \mathrm{~mm}$, $\mathrm{p}=0.724)$. Also, the degree of balloon injury achieved in all groups was comparable (balloon to artery ratio: MeRes-100 $1.10 \pm 0.11$ vs. Absorb BVS $1.11 \pm 0.10, \mathrm{p}=0.893)$. There were no differences in post-implant MLD (MeRes-100 $2.59 \pm 0.26 \mathrm{~mm}$ vs. Absorb BVS $2.76 \pm 0.06 \mathrm{~mm}$, $\mathrm{p}=0.906$ ). Furthermore, both absolute (MeRes-100 $0.12 \pm 0.16 \mathrm{~mm}$ vs. Absorb BVS $0.03 \pm 0.04 \mathrm{~mm}$, $\mathrm{p}=0.150)$ and percentage acute recoil (MeRes-100 
$4.48 \pm 5.90 \%$ vs. Absorb BVS $1.00 \pm 1.43 \%$, $\mathrm{p}=0.128$ ) were comparable and remained at minimal levels in both groups studied. No post-dilatation was performed in any case. Angiographic data at follow-up is summarized in Table 1 . At 2 years $\%$ DS was virtually identical in MeRes-100 when compared to Absorb BVS (MeRes-100 $20.57 \pm$ $\pm 8.93 \%$ vs. Absorb BVS $18.77 \pm 11.78 \%, \mathrm{p}=0.999$ ). Late lumen loss, MLD and RVD were comparable between groups at all time-points. Representative angiographic images over the course of 2 years are presented in Figure 3A.

In vivo OCT analysis. Following device implantation, no differences were observed between MeRes-100 and Absorb BVS in terms of lumen or reference areas (Table 2). Figure 3B illustrates representative OCT images demonstrating the evolution of the MeRes-100 and Absorb BVS integration into the arterial wall and changes in the lumen area over time. At 45 days, both scaffolds showed a $\sim 30 \%$ decrease in lumen area due to neointimal proliferation. Thereafter, the average lumen area increased again towards the baseline value, which was surpassed at 2 years (Fig. 4A). Importantly, in both groups studied lumen area increased at a rate matching the increase in scaffold area (Fig. 4B). Despite small scaffold recoil up to 6 months, the beginning of scaffold expansion process was observed starting at 1 year in both MeRes-100 and Absorb BVS groups. There were no differences in neointimal thickness, neointimal area and \%AS between MeRes-100 and Absorb BVS at any timepoint. Patency ratio increased $\sim 20 \%$ between 1 and 2 years follow up in both groups (Fig. 4C).

\section{Histological analysis}

A summary of the histological analysis is shown in Table 3. Light microscopy revealed that vascular responses to MeRes-100 were comparable with those to Absorb BVS at all time points (Fig. 5). No significant differences were observed in terms of lumen area, \%AS or NIT between the study groups at 30,90 or 180 days. The histological assessment of biocompatibility and safety variables in MeRes-100 and Absorb BVS revealed no differences at any of the studied time points. Virtually all struts were covered in both tested groups at all time-points. Neither MeRes-100 nor Absorb BVS showed evidence of luminal thrombosis in either the main or the side branch of coronary arteries. A slight amount of residual fibrin was observed in both groups at 30 days that gradually decreased and was fully resolved at 180 days. There was numerically greater neointimal inflammation at all time

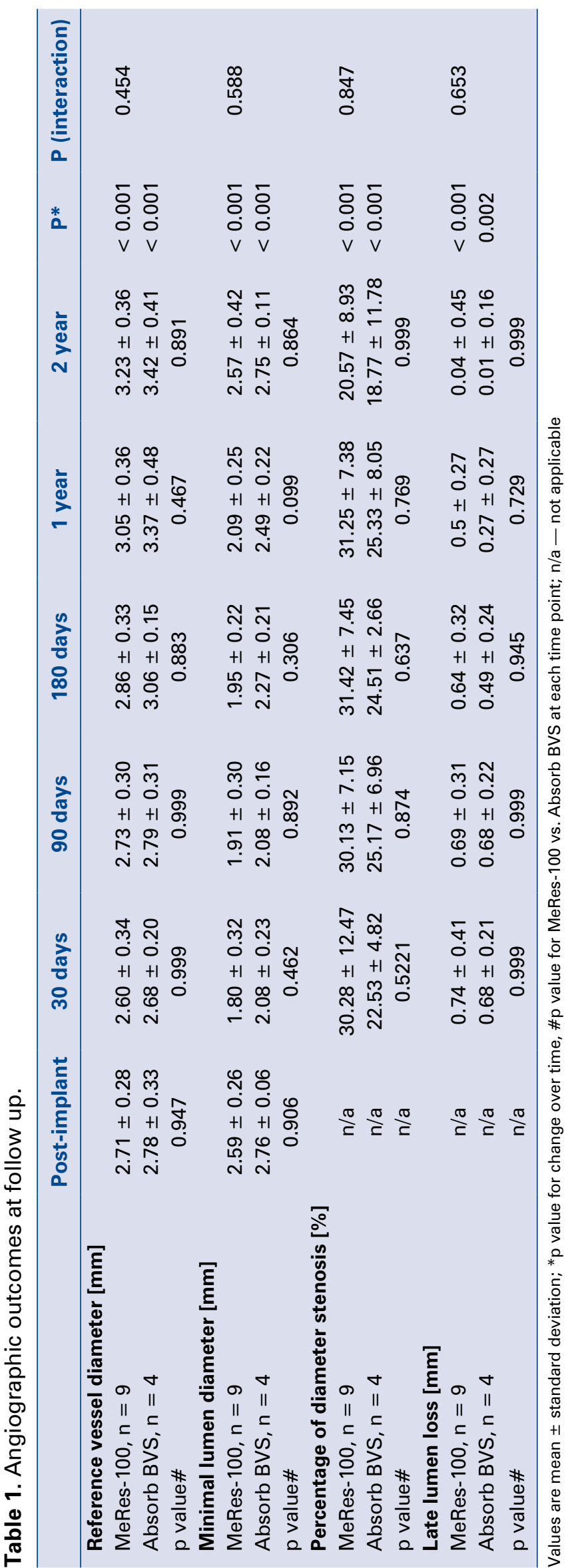




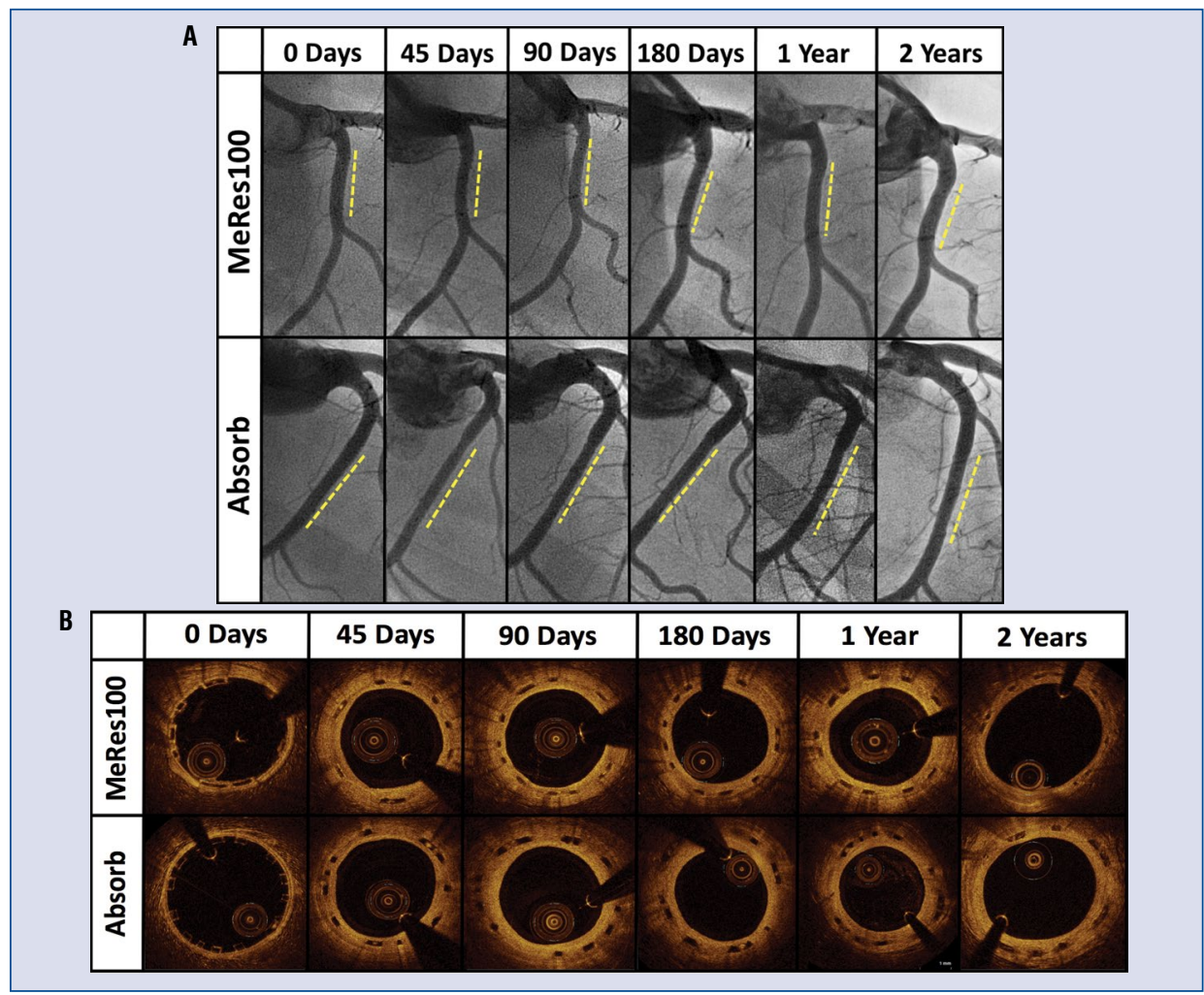

Figure 3. A. Representative angiographic images up to 2 years following scaffold implantation. Both MeRes-100 and Absorb demonstrated decrease of stenosis and late lumen gain between 1 and 2 years after implant; B. Representative images of cross-sectional assessment with optical coherence tomography are shown for MeRes-100 (top panel) and Absorb BVS (bottom panel), demonstrating an increase in lumen area between 1 and 2 years after implant.

points and especially at 180-days in the MeRes-100 group, but did not reach statistical significance because of the small sample size.

\section{Discussion}

In this study, the aim was to evaluate the biocompatibility and vascular healing response of a novel thin-strut PLLA sirolimus-eluting bioresorbable coronary scaffold with hybrid cell design compared to the commercially available Absorb BVS. The major comparative findings of this study in regards to the MeRes-100 are: 1) expansive vascular remodeling starting at 1 year; 2 ) no in vivo scaffold recoil over time; 3 ) adequate inhibition of neointimal proliferation; 4) 6-month healing and inflammatory responses was numerically greater in MeRes-100 as compared to Absorb BVS.

Recently a patient-level, pooled meta-analysis indicated that target vessel-related myocardial infarction was increased with BVS compared with DES, due in part to non-significant increases in periprocedural myocardial infarction and device thrombosis with Absorb BVS [14]. There is also evidence in the literature that hints at an unfavorable peristrut rheology for BVS, as compared with metallic stent [15]. Three-dimensional angiographic reconstruction techniques and computational fluid dynamic data showed low shear stress regions and altered flow patterns in-between BVS struts [16]. It is known that low endothelial shear stress is a powerful local stimulus for atherogenesis, formation, and progression of an early atherosclerotic plaque and differentiation to high-risk plaque [17]. Also, ex-vivo porcine arteriovenous shunt model and a rabbit model of iliofemoral stent implantation demonstrated increased thrombogenicity with respect to platelet aggregation and inflammatory cell adhesion in Absorb BVS when compared to thin strut biodegradable polymer coated DES [18]. Evidence summarized above suggests the necessity in lowering strut thickness without loss of radial strength in order to further improve BRS technology [19]. It is important to highlight that the novel BRS tested in the present study has $100 \mu \mathrm{m}$ strut thickness (over $30 \%$ less that first generation PLLA-based BRS).

Furthermore, optimal BRS design should ensure not only proper acute lumen gain, but also 


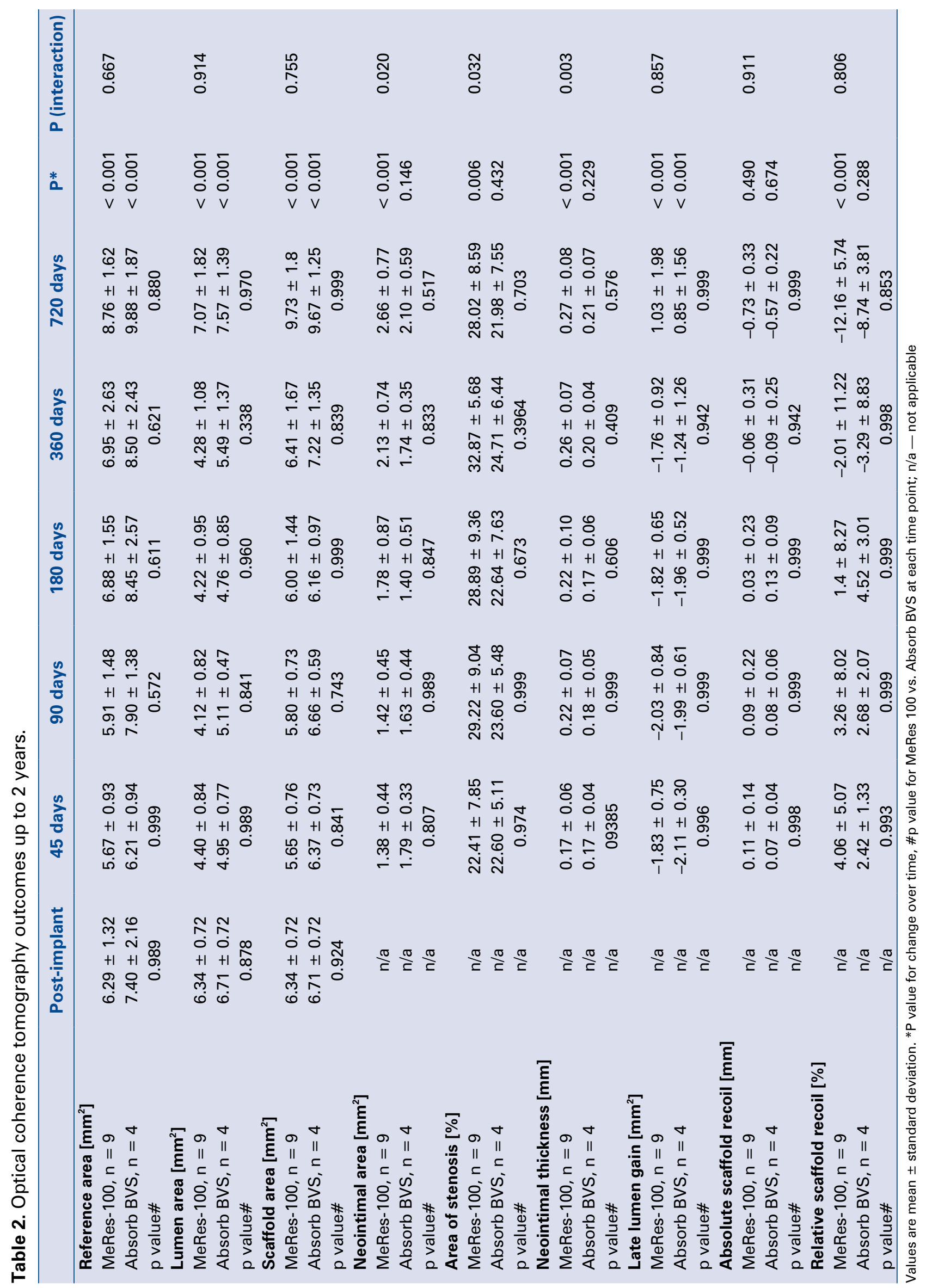




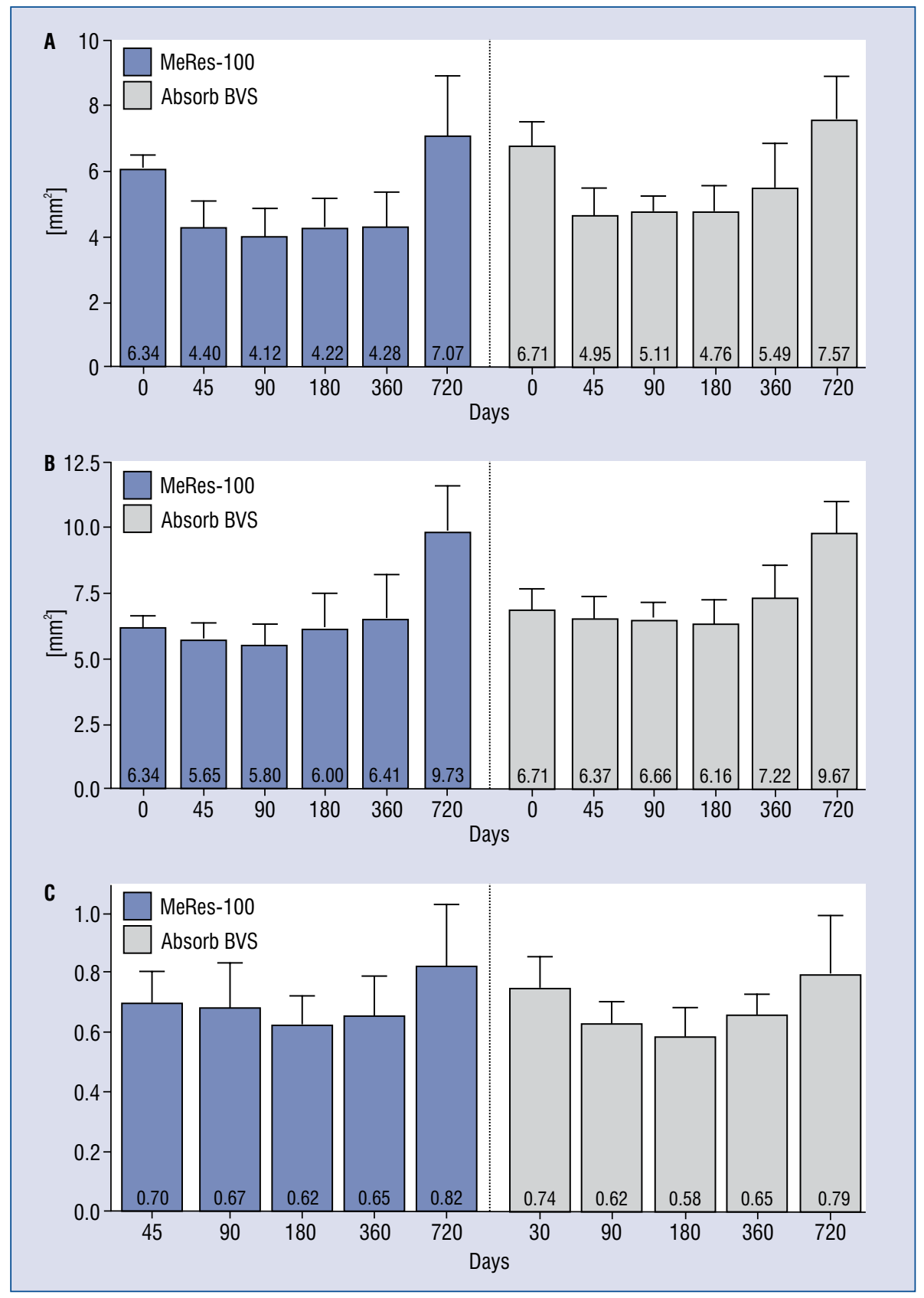

Figure 4. A, B. Lumen and scaffold area over the course of 2 years. Both MeRes-100 and Absorb BVS groups demonstrated increases in both parameters over time after the initial decrease at 45 days; $\mathbf{C}$. The patency ratio in both groups increased over time starting at 180 days.

sustained lumen patency as the vessel heals. Ex vivo degradation and in vivo data of the tested thin strut scaffold demonstrates that radial strength is decreased by $\sim 50 \%$ over 6 months following implantation, which allows complete scaffold resorption in $2-3$ years. Starting at 1-year, an increase in scaffold area was observed in both groups up to 2 years. Then, the in vivo results presented here demonstrate that despite significantly lower strut thickness MeRes-100 displays similar post-proce- dural biomechanical performance with long-term architectural stability compared to Absorb BVS. Also being studied were in vivo changes of the vessel architecture after implantation of the scaffolds studied. The data demonstrates that the lumen area of reference segments increased over time, reflecting normal growth of the animal. Importantly, the patency ratio of BRS treated vessels increased over time and reached $\sim 0.8$ at 2 years follow up in both devices (Fig. 4C). This demonstrates that 


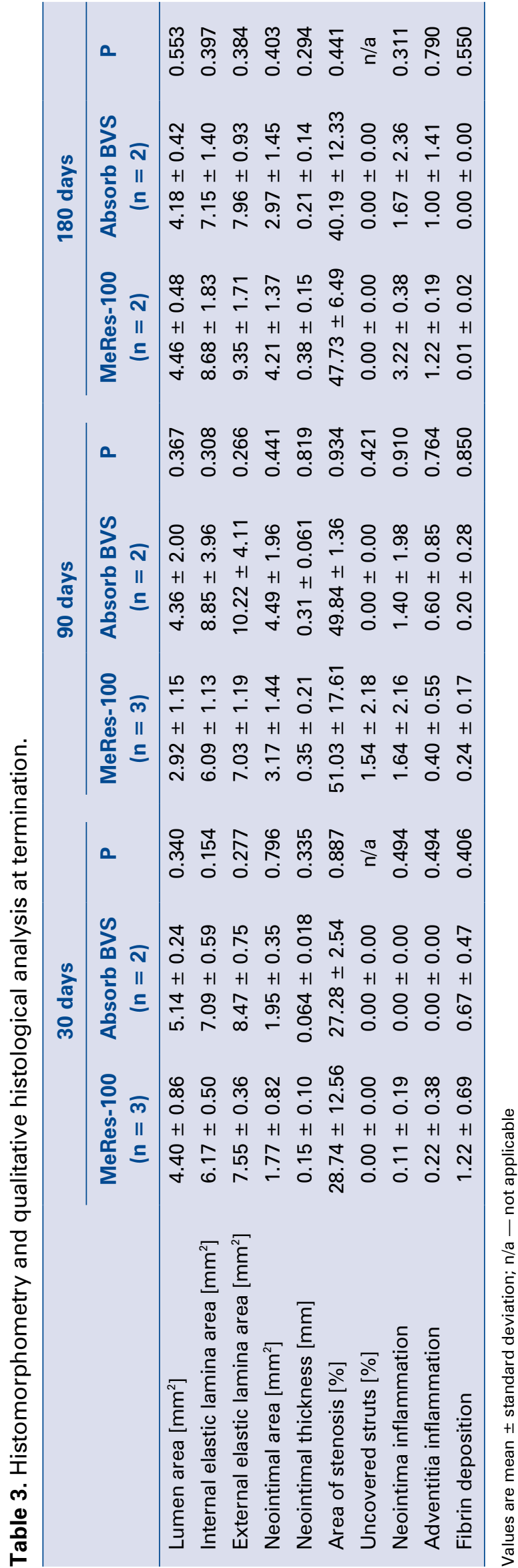

both scaffolds studied accommodated natural late growth of arteries in this model.

In vivo pharmacokinetic studies have reported that in pig models, systemic peak levels of sirolimus reach $2.63 \mathrm{ng} / \mathrm{mL}$ at $\sim 1 \mathrm{~h}$ after sirolimus-eluting stent implantation [20] and by day 29 only $21 \%$ of the initial sirolimus concentration remained on the stent and only minimally detectable systemic drug levels were present beyond 7 days [21]. In the present study, the peak concentration of sirolimus in blood occurred at 1-4 h post implant and the average of maximum concentration of sirolimus was $7.38 \pm 0.42 \mathrm{ng} / \mathrm{mL}$. The blood levels of sirolimus quickly declined to below the limit of quantification after 14 days post MeRes-100 implantation. Arterial tissue concentrations gradually declined over time and were still detectable 186 days following device deployment.

An important objective of this study was the evaluation of vascular healing following the implantation of the novel thin strut BRS. Histology results, although limited to 180 days, demonstrated that MeRes-100 had comparable healing responses to Absorb BVS at all time points, although the lumen area was smaller at 30 and 90 days in MeRes-100 but was similar at 180 days. Whereas, inflammation was greater in MeRes-100 at all time points, especially at 180 days, but the number of implants at each time point was inadequate to draw any definite conclusions. The slightly higher levels of neointimal inflammation seen in the MeRes-100 group at 180 days are likely related to the degradation process already underway, which is supposed to be faster than that of the Absorb BVS. The levels of fibrin deposits in both BRS groups were highest at 1-month. After that time point, drug release slows down leading to fibrin deposit absorption. Also, there were no significant differences in NIT or \%AS in the groups studied at any time point. These findings suggest that MeRes-100 may have the potential to improve performance of current generation BRS by providing a relatively biocompatible and mechanically durable platform at over $30 \%$ lower strut thickness than first generation PLLA-based BRS.

\section{Limitations of the study}

The present study has some limitations that are important to note. First, the study was performed in healthy coronary arteries in the swine model of restenosis. All scaffolds were implanted in the main coronary artery segments avoiding large side branches $(>2.0 \mathrm{~mm})$ and the number of implants at each time point are too few to draw any definite conclusion. The findings cannot predict 


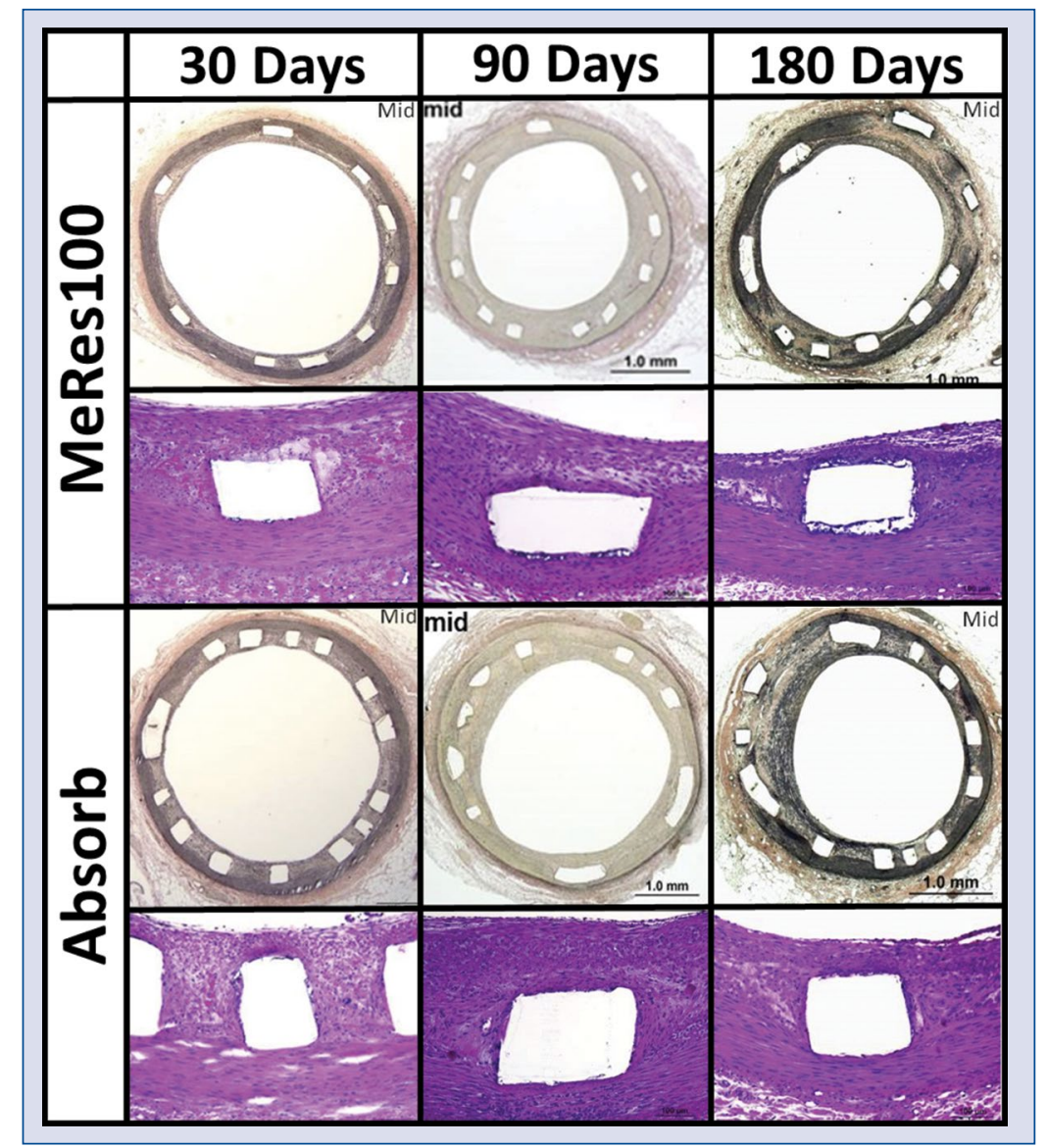

Figure 5. Representative histological images of the MeRes-100 and Absorb BVS in porcine coronary arteries at 1,3 and 6 months after implantation.

its clinical performance among patients with high atherosclerotic burden and especially by lessons learnt from ABSORB III clinical trial. However, preliminary clinical data with MeRes- 100 revealed favorable safety and efficacy in a non-randomized multicenter first-in-human study [22]. On the other hand, some numerical differences between both scaffolds might not have reached statistical significance due to small sample size. Further, this study includes only 30-, 90- and 180-day histological data only. The reported study is part of a larger long-term biocompatibility study and therefore all the animals continued to undergo imaging, and eventually late histopathologic follow up. The 30-, 90- and 180-day interim data provide a snapshot of the healing profile, but long-term histological data are needed.

\section{Conclusions}

In conclusion, data herein indicates that MeRes-100 scaffold used in the present study appeared comparable to control Absorb BVS in terms of biomechanical performance and vascular healing response up to 180 days, and the OCT study up to 2 years in healthy porcine arteries also showed similarity to Absorb BVS. Treated arterial segments underwent vascular remodeling in parallel with the degradation process of the tested polymer scaffolds. Also, pharmacokinetic analysis demonstrated the presence of sirolimus in arterial tissue 180 days following scaffold implantation. The scaffold examined in this preclinical study is currently used in clinical studies worldwide, as well as being commercially available in select geographies. Therefore, continuation of its long-term preclinical safety and biocompatibility evaluation is of paramount clinical importance.

Funding: This study was funded by Meril Life Sciences Pvt. Ltd.

Conflict of interest: None declared 


\section{References}

1. Onuma Y, Ormiston J, Serruys PW. Bioresorbable scaffold technologies. Circ J. 2011; 75(3): 509-520, indexed in Pubmed: 21301138.

2. Ellis SG, Kereiakes DJ, Metzger DC, et al. Everolimus-Eluting Bioresorbable Scaffolds for Coronary Artery Disease. N Engl J Med. 2015; 373(20): 1905-1915, doi: 10.1056/NEJMoa1509038, indexed in Pubmed: 26457558.

3. Serruys PW, Chevalier B, Dudek D, et al. A bioresorbable everolimus-eluting scaffold versus a metallic everolimus-eluting stent for ischaemic heart disease caused by de-novo native coronary artery lesions (ABSORB II): an interim 1-year analysis of clinical and procedural secondary outcomes from a randomised controlled trial. Lancet. 2015; 385(9962): 43-54, doi: 10.1016/S01406736(14)61455-0, indexed in Pubmed: 25230593.

4. Serruys PW, Chevalier B, Sotomi Y, et al. Comparison of an everolimus-eluting bioresorbable scaffold with an everolimus-eluting metallic stent for the treatment of coronary artery stenosis (ABSORB II): a 3 year, randomised, controlled, single-blind, multicentre clinical trial. Lancet. 2016; 388(10059): 2479-2491, doi: 10.1016/ S0140-6736(16)32050-5, indexed in Pubmed: 27806897.

5. Kereiakes DJ, Ellis SG, Metzger C, et al. ABSORB III Investigators. 3-Year Clinical Outcomes With Everolimus-Eluting Bioresorbable Coronary Scaffolds: The ABSORB III Trial. J Am Coll Cardiol. 2017; 70(23): 2852-2862, doi: 10.1016/j. jacc.2017.10.010, indexed in Pubmed: 29100702.

6. Otsuka F, Cheng Qi, Yahagi K, et al. Acute Thrombogenicity of a Durable Polymer Everolimus-Eluting Stent Relative to Contemporary Drug-Eluting Stents With Biodegradable Polymer Coatings Assessed Ex Vivo in a Swine Shunt Model. JACC Cardiovasc Interv. 2015; 8(9): 1248-1260, doi: 10.1016/j. jcin.2015.03.029, indexed in Pubmed: 26292590.

7. Koskinas KC, Chatzizisis YS, Antoniadis AP, et al. Role of endothelial shear stress in stent restenosis and thrombosis: pathophysiologic mechanisms and implications for clinical translation. J Am Coll Cardiol. 2012; 59(15): 1337-1349, doi: 10.1016/j. jacc.2011.10.903, indexed in Pubmed: 22480478.

8. Azzalini L. L'Allier PL. Bioresorbable vascular scaffold thrombosis in an all-comer patient population: single-center experience. J Invasive Cardiol. 2015; 27: 85-92.

9. Onuma Y, Serruys PW, Gomez J, et al. Comparison of in vivo acute stent recoil between the bioresorbable everolimus-eluting coronary scaffolds (revision 1.0 and 1.1) and the metallic everolimus-eluting stent. Catheter Cardiovasc Interv. 2011; 78(1): 3-12, doi: 10.1002/ccd.22864, indexed in Pubmed: 21413120.

10. Cheng Y, Gasior P, Shibuya M, et al. Comparative characterization of biomechanical behavior and healing profile of a novel ultrahigh-molecular-weight amorphous poly-l-lactic acid sirolimuseluting bioresorbable coronary scaffold. Circ Cardiovasc Interv. 2016; 9(10), doi: 10.1161/CIRCINTERVENTIONS.116.004253, indexed in Pubmed: 27694138.

11. Gomez-Lara J, Brugaletta S, Diletti R, et al. A comparative assessment by optical coherence tomography of the performance of the first and second generation of the everolimus-eluting bioresorbable vascular scaffolds. Eur Heart J. 2011; 32(3):
294-304, doi: 10.1093/eurheartj/ehq458, indexed in Pubmed: 21123276.

12. Strandberg E, Zeltinger J, Schulz DG, et al. Late positive remodeling and late lumen gain contribute to vascular restoration by a non-drug eluting bioresorbable scaffold: a four-year intravascular ultrasound study in normal porcine coronary arteries. Circ Cardiovasc Interv. 2012; 5(1): 39-46, doi: 10.1161/CIRCINTERVENTIONS.111.964270, indexed in Pubmed: 22253358.

13. Yazdani SK, Pacheco E, Nakano M, et al. Vascular, downstream, and pharmacokinetic responses to treatment with a low dose drug-coated balloon in a swine femoral artery model. Catheter Cardiovasc Interv. 2014; 83(1): 132-140, doi: 10.1002/ccd.24995, indexed in Pubmed: 23703778.

14. Stone GW, Gao R, Kimura T, et al. 1-year outcomes with the Absorb bioresorbable scaffold in patients with coronary artery disease: a patient-level, pooled meta-analysis. Lancet. 2016; 387(10025): 1277-1289, doi: 10.1016/S0140-6736(15)01039-9, indexed in Pubmed: 26825231.

15. Tenekecioglu E, Torii R, Bourantas C, et al. Preclinical assessment of the endothelial shear stress in porcine-based models following implantation of two different bioresorbable scaffolds: effect of scaffold design on the local haemodynamic micro-environment. EuroIntervention. 2016; 12(10): 1296, doi: 10.4244/ EIJY16M05_01, indexed in Pubmed: 27180301.

16. Gogas BD, King SB, Timmins LH, et al. Biomechanical assessment of fully bioresorbable devices. JACC Cardiovasc Interv. 2013; 6(7): 760-761, doi: 10.1016/j.jcin.2013.04.008, indexed in Pubmed: 23866188.

17. Chatzizisis YS, Coskun AU, Jonas M, et al. Role of endothelial shear stress in the natural history of coronary atherosclerosis and vascular remodeling: molecular, cellular, and vascular behavior. J Am Coll Cardiol. 2007; 49(25): 2379-2393, doi: 10.1016/j. jacc.2007.02.059, indexed in Pubmed: 17599600.

18. Koppara T, Cheng Qi, Yahagi K, et al. Thrombogenicity and early vascular healing response in metallic biodegradable polymerbased and fully bioabsorbable drug-eluting stents. Circ Cardiovasc Interv. 2015; 8(6): e002427, doi: 10.1161/CIRCINTERVENTIONS.115.002427, indexed in Pubmed: 26022535.

19. Kang J, Han JK, Yang HM, et al. Bioresorbable vascular scaffolds: are we facing a time of crisis or one of breakthrough? Circ J. 2017; 81(8): 1065-1074, doi: 10.1253/circj.CJ-17-0152, indexed in Pubmed: 28680011.

20. Suzuki T, Kopia G, Hayashi S, et al. Stent-based delivery of sirolimus reduces neointimal formation in a porcine coronary model. Circulation. 2001; 104(10): 1188-1193, indexed in Pubmed: 11535578 .

21. Vetrovec GW, Rizik D, Williard C, et al. Sirolimus PK trial: a pharmacokinetic study of the sirolimus-eluting Bx velocity stent in patients with de novo coronary lesions. Catheter Cardiovasc Interv. 2006; 67(1): 32-37, doi: 10.1002/ccd.20565, indexed in Pubmed: 16342216.

22. Seth A, Onuma Y, Costa R, et al. First-in-human evaluation of a novel poly-L-lactide based sirolimus-eluting bioresorbable vascular scaffold for the treatment of de novo native coronary artery lesions: MeRes-1 trial. EuroIntervention. 2017; 13(4): 415-423, doi: 10.4244/EIJ-D-17-00306, indexed in Pubmed: 28504218. 